\title{
Wearable Bits
}

\author{
Scaffolding Creativity with a Prototyping Toolkit for Wearable E-textiles
}

\author{
Lee Jones, Sara Nabil, Amanda McLeod, Audrey Girouard \\ Carleton Universiy \\ Ottawa, Ontario, Canada \\ \{Lee.Jones, Sara.Nabil, Amanda.McLeod, Audrey.Girouard\}@carleton.ca
}
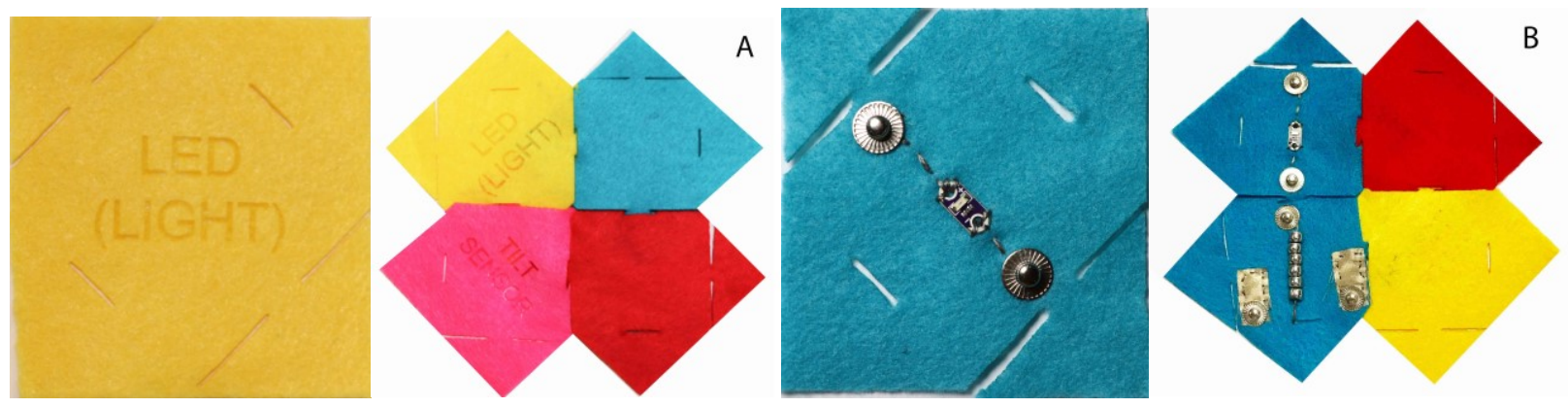

Figure 1-Participants built wearable e-textile prototypes with (a) low-fidelity Wearable Bits and (b) medium-fidelity Wearable Bits

\begin{abstract}
Smart garment and wearable e-textile prototypes are difficult to co-design because of the variety of expertise needed (garment design, sewing skills, hardware prototyping, and software programming). To help with this, we developed a toolkit for prototyping wearable etextiles, named Wearable Bits, which enables co-design with non-expert users without demanding sewing, hardware or software skills. We developed a low-fidelity and medium-fidelity experience prototype of the toolkit and ran a series of workshops where non-expert users designed their own e-textile wearables using Wearable Bits. In this paper, we discuss the ideas they developed, their construction techniques, the roles individuals took on while building, and suggestions for future toolkits.
\end{abstract}

\section{CCS CONCEPTS}

- Human-centered computing $\rightarrow$ User interface toolkits

\section{KEYWORDS}

e-textile; smart clothing; wearable; constructive assembly; toolkit; fidelity; prototype; co-design

\footnotetext{
Permission to make digital or hard copies of all or part of this work for personal or classroom use is granted without fee provided that copies are not made or distributed for profit or commercial advantage and that copies bear this notice and the full citation on the first page. Copyrights for components of this work owned by others than ACM must be honored. Abstracting with credit is permitted. To copy otherwise, or republish, to post on servers or to redistribute to lists, requires prior specific permission and/or a fee. Request permissions from Permissions@acm.org.

TEI '20, February 9-12, 2020, Sydney, NSW, Australia

(c) 2020 Association for Computing Machinery.

ACM ISBN 978-1-4503-6107-1/20/02 \$15.00

https://doi.org/10.1145/3374920.3374954
}

\section{ACM Reference format:}

Lee Jones, Sara Nabil, Amanda McLeod, Audrey Girouard. 2020. Wearable Bits: Scaffolding Creativity with a Prototyping Toolkit for Wearable Etextiles. In Proceedings of the Fourteenth International Conference on Tangible, Embedded, and Embodied Interaction, February 9-12, 2020, Sydney, Australia. ACM.

\section{INTRODUCTION}

Hardware products are often risky because unlike software where a company can provide updates, hardware is difficult to change after purchase. Co-designing hardware products with consumers, and involving them right from the beginning, can help reduce this risk by ensuring that the final product fits users' needs [44]. Smart garments and wearable e-textiles would benefit from co-design in the same manner. However, this process is challenging because it requires a wide variety of expertise including, but not limited to, garment design, sewing and tailoring skills, hardware prototyping, and software programming. On top of this, having electronics in your clothes is still a foreign concept to most users, with the exception of a few available consumer products limited to health and fitness.

To help with the co-design of these e-textile garments, we have created 'Wearable Bits', a toolkit for designing wearable e-textile prototypes. Wearable Bits is made up of felt fabric pieces cut out using modular laser-cut tessellations. Individual pieces include plain, sensing and actuating pieces, which all can be attached, aggregated and disassembled as needed to create prototypes for e-textile 
garments of any form or size. Wearable Bits draws upon Tangible Bits where individuals can "grasp and manipulate" bits merging the physical with the digital, and in particular the constructive assembly toolkit approach $[20,22]$. We developed both low fidelity and mediumfidelity versions of our toolkit to evaluate both of them for effective co-design (see Figure 1) and to provide an "experience prototype" [4] of the envisioned system. The low-fidelity toolkit has sensors and actuators written out as words on the felt pieces, whereas the medium-fidelity toolkit has interactive components made with machine sewn, hand sewn, and embroidered conductive and resistive threads. This paper provides the necessary first step of discovering how users would interact and build with such a toolkit before developing fully wearable bits. We conducted a series of workshops with 13 different groups to explore how non-experts would use Wearable Bits to codesign for their own needs.

The findings of our study provide insights to e-textile prototyping methods and co-design with non-expert users. Our key contributions in this paper are:

1. Designing a low and medium fidelity version of a skillsfree toolkit for rapid-prototyping of wearables.

2. Empowering non-expert users to design e-textiles for their daily activities and individual needs.

3. Evaluating how this type of modular tangible toolkit could impact the types of wearable prototypes individuals decide to design and how they design them.

\section{RELATED WORK}

Scaffolds within the field of tangible interaction are props or tools we can use to help us think through problems [18] and document and externalize those thoughts [16]. In the area of wearables and e-textiles, there are many different types of scaffolds that help users come up with wearable concepts, express preferences, and build wearable prototypes. Scaffolds are especially important for co-design and are often called "co-design toolkits" in how they enable individuals who likely have not designed before to express their ideas [44]. These co-design toolkits can vary from craft supplies, ideation cards, to constructive assemblies [44]. For wearable e-textiles, we see two types of scaffolding toolkits: those that are cognitive scaffolds, mostly used for wearable ideation, and those that are embodied scaffolds where individuals can develop "experience prototypes" [4] of their wearable concepts.

\subsection{Cognitive Scaffolds for Wearables}

A cognitive scaffold is a tool that helps people cognitively think through a problem [47]. In the realm of wearables, ideation activities and samples can help end users come up with wearable concepts through inspiration. Cardsorting is an activity that involves cards with different clothing characteristics, interactions, and garment types and having users organize them based on their preferences [34]. This method can be useful at the beginning of the design process for helping users in divergent thinking and imagining nonstandard smart garments (i.e., thinking beyond smart shirts). E-textile swatchbooks contain sample swatches and are typically used for knowledge sharing [15, 39]. Swatchbooks are useful for co-design because they allow users, who are unlikely to have experience with e-textiles, to explore their potential and come up with wearable concepts $[12,51]$. Sketching wearable concepts with notes or drawings is a way of externalizing wearable concepts for sharing ideas and receiving feedback [52]. These cognitive scaffolds are useful for coming up with new ideas and sharing them, but because the concepts are not worn or presented at life size, the subtleties of what it is like to have or to wear such a garment in everyday life still needs to be explored.

\subsection{Embodied Scaffolds for Wearables}

Experience prototypes allow individuals to experience a prototype for themselves [4]. So rather than just thinking about a concept through sketches or swatches, an experience prototype simulates the feeling one might have with a wearable by wearing it. These tangible prototypes are useful because they emulate how we interact with and understand our world $[20,22]$ as we use our body's senses to evaluate our prototype [47]. People can build the prototypes on themselves to "ideate on the body" and "enact" what it would be like to wear them [49]. These embodied prototypes then help people to move from an abstract concept to a concrete idea and to iteratively improve upon it [18]. They are especially useful when codesigning with individuals from different fields since they leverage their physical skills $[11,30,46]$. They can then use these physical traces to express ideas to their teammates and keep a record of their insights $[18,30,46]$.

The most common example of an experience prototype is a low-fidelity prototype, which is often developed with simple craft materials [34]. The benefit of this approach is that users can "try on" their prototypes and experiment with different scenarios, without needing to know how to build the technology. 
Constructive assemblies are tangible user interfaces that can be constructed, deconstructed, and constructed again [20, 22, 33]. This feature makes them useful for co-design because users can try out ideas and change their prototype as they go [14]. The potential added value when comparing constructive assemblies to low-fidelity prototypes is that users can also try out and prototype the interactions, but this also can add limitations to creativity depending on the design of the kit. One common constructive assembly used for co-design is LittleBits [1], which has a series of chained components that connect with magnets. Because of their hard components they tend to be used for co-designing accessories (e.g. MakerShoe [28]). MakerWear [27] supports making wearable prototypes using hard components that clip onto a mesh. Because these toolkits have chained components this often limits interaction to one area of the body. LilyPad [5, 6, 8] is a sewable Arduino toolkit for electronic textiles. Conductive thread can be used to sew the kit together and thus enable spreading components across the body. The LilyPad is limited as a constructive assembly because taking out stitches and resewing is destructive to most fabrics, but the toolkit is still a robust prototyping tool with a wide variety of components. This is demonstrated in the several constructive assemblies that are extensions of the LilyPad toolkit such as Craftec [23] (which adds magnetic connections) and Patchwork [3] (which uses sewing snaps). Both the LilyPad and a similar kit $I^{*}$ Catch [36] have hardware boards for the individual components rather than soft sensors. Quilt Snaps [7] is a series of soft decorative patches that can be connected together with sewing snaps, but the square pattern hinders their application to wearable garments. The TeeBoard [35] is a t-shirt that acts as a breadboard with the same sewing snap connections, but is limited to t-shirt prototyping. A Kit-of-No-Parts [38] is a type of "un-toolkit" where individual soft sensors and components are handmade with craft materials. The kit has a large amount of flexibility, but as an "un-toolkit" there is not a systematic way of connecting the individual components.

Previous work on prototyping e-textiles is mainly limited to expert designers [38] or introduced to other professional designers [10,13]. Limited work has engaged end-users or empowered them with easy-to-use fabric toolkits. Online tutorials of e-textiles require users to possess crafting and making skills such as sewing [29, 41], electronics expertise [32] and software programming [6]. Toolkits for beginners often focus on education and are used as teaching tools to give beginners those necessary skill sets. Such accounts leave out non-expert users that may lack some or part of these skills but would still contribute to co-design of etextile wearables that support them in their daily needs. This research gap limits the accessibility of e-textile making.

\section{WEARABLE BITS}

We wanted to develop a co-design toolkit that easily transitions from cognitive scaffold to embodied scaffold using fabric cards and swatches that can then connect to be used for prototyping e-textiles on the body.

\subsection{The Toolkit}

Wearable Bits [24] are made of modular laser-cut tessellations of $160 \mathrm{~g}$ acrylic felt fabric. We chose felt because it is a non-precious material often used for crafts, therefore ideal for early-stage prototypes. The bit modules can be manually interlocked together by inserting the arrows into the slots. This type of modular textile design has been used for aesthetic purposes [19, 26], taught as a method to reduce textile waste [9], and is also useful for codesign applications because of the ability to assemble and disassemble pieces [37]. We optimized the arrow and slot tab design used by Soepboer et al. [2] with narrower slots so that the fabric would hold together like a garment and withstand weight. The surface areas can then be used for circuit components. We included four sizes of Wearable Bits, small (3" x 3"), medium (4 1/4” x 4 1/4"), large (6 1/4” x 6 $1 / 4$ "), extra-large (13 $1 / 2 "$ x $61 / 4 ")$ to enable flexibility, customizability and rapid prototyping (Figure 2).

There are two types of Wearable Bits-low-fidelity and medium-fidelity. Low-fidelity Wearable Bits have the name of the component laser cut into the felt. The idea is that they act similar to the ideation cards used in card sorting except in this case participants can put them on and decide where items should be placed. Medium-fidelity components are made by embroidering, sewing, hand stitching and felting conductive and resistive threads and fibers into the felt components. The touch matrix, slider, pinch sensor, and button all use embroidery patterns developed from Sketch\&Stitch [13]. The tilt sensor, stretch sensor, potentiometer, stroke sensor, pressure sensor and speaker are hand sewn patterns from A Kit-of-No-Parts [38]. The heat and colour change components are made with resistive thread, with thermochromic fabric on top for colour change. The LED is from the LilyPad kit and the vibration motor from an Arduino kit. Metal snaps are punched into the fibers to make connections between different swatches in future higher-fidelity versions, but we could also use safety pins or conductive fabric to make the connections. We also provided sewing accessories such as buttons, 
A)

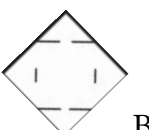

B)

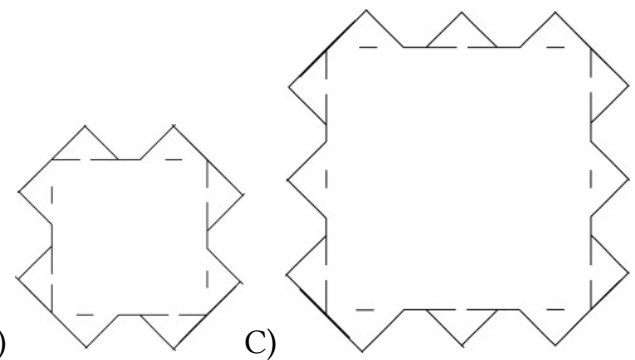

D)

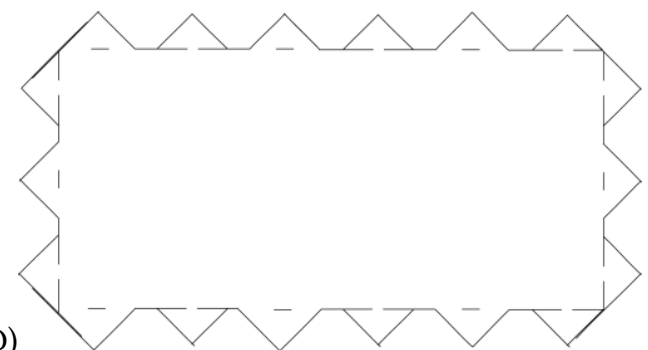

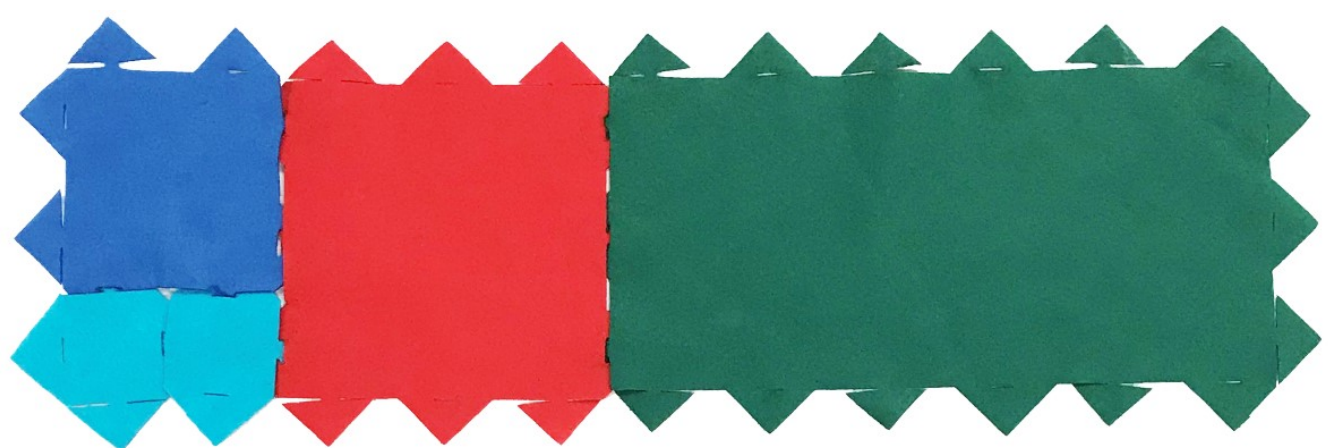

Figure 2 - The four sizes of Wearable Bits can all be connected (A) small (B) medium (C) large (D) extra-large.
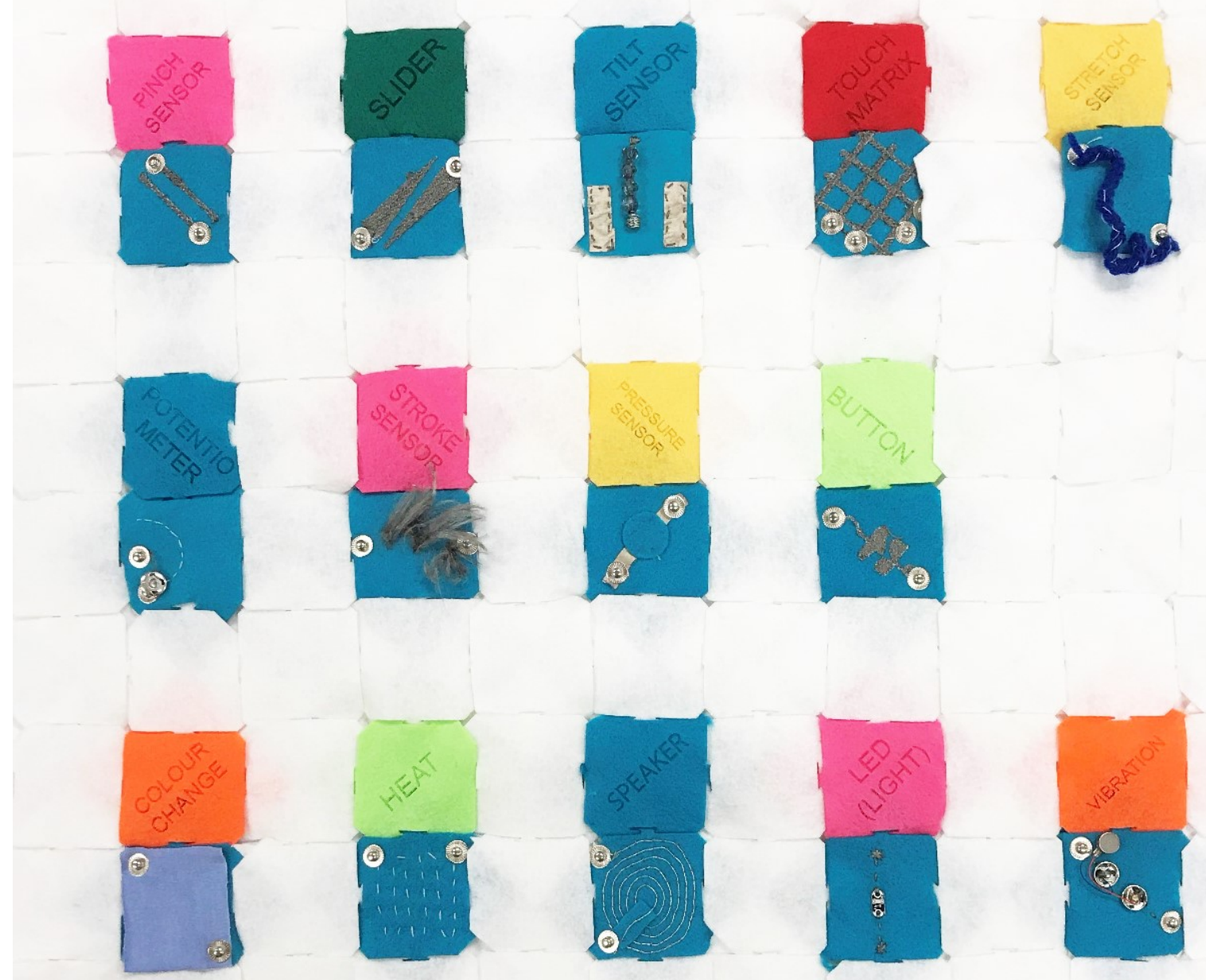

Figure 3 - The low and medium-fidelity Wearable Bits 
zippers, clasps, so that participants could prototype not only the design of the textile but also the design of closures. For the components, we wanted to focus on ones that participants were not likely to be aware of, such as beading to make a tilt sensor, or fringe for a stroke sensor, but participants were also invited to add swatches to the toolkit by writing sensor or actuator names onto blank bits.

\subsection{Method}

To evaluate the kit, our study had pairs of participants go through ideation activities to come up with many wearable concepts, come together to decide on their favourite that they would like to build, and then build that prototype with the toolkit. We ran a series of 13 workshops with pairs of individuals who knew each other before the study. The recruitment process encouraged those with 'no prior experience with e-textiles' to sign up. We recruited 26 participants from varying age groups ( 9 aged 18-30, 12 aged 31-60, and 5 aged 61-90) and genders (15 women, 10 men and 1 non-binary). The first seven workshops were conducted using low-fidelity Wearable Bits while the last six workshop sessions were conducted using mediumfidelity Wearable Bits.

In each workshop session, the study began by giving each participant a demographic questionnaire about any previous experience with e-textiles and garment construction. We then gave a priming presentation on etextiles where we explained the components of the toolkit and showed them example images of what the components look like as swatches. Then participants had the opportunity to brainstorm ideas for their own garment. To help with this, we prepared a brainstorming activity where participants picked cards from four piles and came up with eight different wearable e-textile concepts using a design sprint technique called Crazy 8s [31] (Figure 4). The four piles were types of garments, types of situations or activities, adverbs to describe the interaction, and who the interaction of the garment was for (see Figure 4). For example, a person could pick a card for a jacket (garment), for the office (situation), for self-expression (for who), and regularly (adverb to describe the interaction). We put a timer on for 8 minutes to help encourage them to focus on the quantity of ideas they could produce rather than evaluating their ideas at this stage. In total, they would try to pick 8 sets of cards to make 8 wearable e-textile concepts.

After 8 minutes teams then picked their favourite idea and wrote a sentence about their concept which could be updated as they went through the session. They then picked one partner to be have the "wearer" role (meaning they

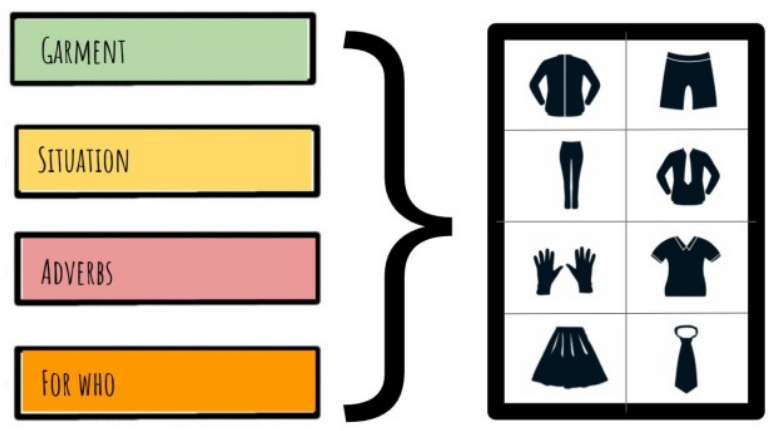

Figure 4 - Brainstorming activity: individuals grab ideation cards and then draw a concept.

would wear the prototype) and the other the "non-wearer" role (meaning they would not wear the prototype). The only rules provided to them were that they needed to consider how the garment would be taken on and off, and how the garment would be turned on and off. Participants had approximately 45 minutes to build their prototype. Once they completed prototyping, they "presented" their prototype and were video recorded while the researcher asked any clarifying questions about their prototype. At the end of all the sessions participants completed a post-study questionnaire asking about their experience using the toolkit.

\subsection{Data Analysis}

Our gathered data included the drawings and notes from their brainstorming sessions, our field notes and observations during the prototyping process, their filled questionnaires and video recordings of their presentations. We transcribed all the video recordings, and performed a thematic analysis on both the transcriptions and observational notes. We chose thematic analysis to capture the variety of reoccurring codes and general themes over different groups, as well as to delve into the social dynamics that occur when people are designing and building together. Participants were grouped in pairs and we identify these as G1 through to G13 with W to identify 'wearers' and NW to identify 'non-wearers'.

\section{FINDINGS}

During thematic analysis, we found five main themes in the participants' prototypes and how they constructed them. Here we discuss their wearable e-textile concepts, their construction techniques, their roles, how they built "onthe-fly", and aesthetics.

\subsection{The Land of Opportunity}

By analyzing the brainstorming and ideation carried out by each of our 13 groups, we found that their designs of 
interactive garments with Wearable Bits were mainly to seize one of three opportunities: 1) garments for well-being, 2) garments for comfort, and 3) garments for selfexpression.

Garments for well-being enabled participants to design for their psychological and physiological daily needs. G2 made a "cheer-you-up-dress" and measures mood through posture and plays gentle music to cheer you up and encourage you to get dancing. G3 made a hat for individuals with memory impairments and their carers to help them feel more comfortable going outside. In fact, several garments were designed to measure body signals and then cued the wearer through actuation to do an action. G6 made a sleeping vest that measured circadian rhythms (through body temperature changes) and cued the child that it is time for bed. G7 made running shorts that signaled to the wearer that they need to pick up their pace. G8 made a sleeve for people with arthritis that measured temperature and then encouraged them to get moving to increase blood flow.

Garments for comfort enabled participants to improve the wearer's current state and support their personal comfort. G1 made a shirt that the wearer can turn into a crop top when they feel warm and this action also actuates cooling pads under their armpits to cool them down. G5 made a hat for cold weather that warms up, plays music in the earmuffs, and lights up if you are walking at night. G11 made a creativity cape that allows them to avoid distractions while working by changing the temperature, changing the music, and creating more light for working all within the cape.

Garments for self-expression enabled people to communicate with those around them through their clothing, including garments for musical and dance performance, activism and public awareness. G4 made a rocker jacket that would generate music as the performer danced. G9 made a modular sleeve that would visualize the music during a dance performance through light and colour change both to augment the performance and to be more inclusive of individuals who have hearing impairments. G10 and G13 made garments that outwardly expressed body signals. G10 made a shirt for going out dancing that lit up as your temperature increased. G13 made a shirt that someone could wear throughout a pregnancy that would change colour as their belly grew and would light up when there was a kick. G12 made a modular activism wearable that aims to make individuals aware of invisible surveillance from infrared (IR) sensor and radio-frequency identification (RFID) sensors that are common in public places. The wearable lights up when it detects these sensors with the aim of encouraging discussion with the public.

Garments at times incorporated two or more categories. For example, the G2 cheer-you-up-dress, though its main purpose was to improve the wearer's well-being, the music had an aspect of performance. G2 W envisioned outsiders becoming curious about the dress, "So it'd be like "Where is that sound coming from?" and you'd be like [whispers] "It's that girl's dress" [pointing]."

\subsection{Construction Before Interaction}

Many of the groups had similarities among their construction techniques for building their wearable prototype. There was a tendency to build the garment first with the large and extra-large squares. This occurred in 10 of the 13 groups. Participants mentioned they used the larger squares to shorten the amount of time it would take to build the garment. G6 used larger pieces "because it was faster to put together" and G7 and G10 used the technique to "save on time". Participants also used larger pieces first because of the scale of the garment their group wanted to make. G7 "needed something big to cover some leg area" and G11 "was thinking cape so I was thinking big and that just led to the bigger pieces". The other three groups who did not use this technique made smaller accessories and worked with the small and medium squares.

Structurally many groups decided to build their garment first with plain pieces and then add the interactive components second. In some cases, these components were treated as different layers of the garment. Seven groups glued, taped or pinned their interactive components on top of the garment structure instead of using the interlocking tabs. G7 "For things we didn't weave together we just glued them on." G13 "In terms of construction we started off with the big pieces first and then built down to the details where things would kind of go and sort of stuck them there". Another technique used for smaller accessories was to weave the interactive components together to make a second top layer above the bottom structure. This technique was applied to two hat prototypes.

For the groups that wove their interactive components into their garment they often placed these components along the edges of larger pieces. These interactive components then acted as "connectors". G1 "For the main part it was the bigger one, and then for connecting them we have the smaller one" and G10 "for the areas we wanted to focus on we used the smaller ones in the creases, so the LEDs and stuff like that". The smaller squares were also used to close the garment. G2 "We actually tended to use the smaller 


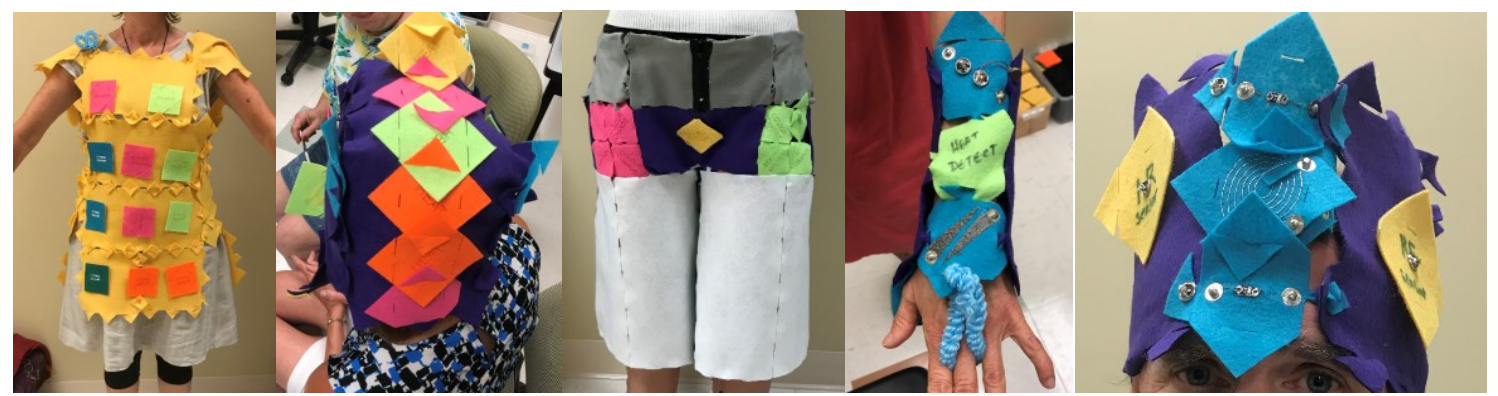

Figure 5-Examples of different construction techniques from left to right (a) gluing individual pieces onto the garment structure (b) weaving the interactive pieces as a second top layer (c) fully integrating them into the garment and gluing the seams down (d) weaving them into the garment (e) using pins and glue to attach the interactive components.

pieces for the fastening at the side which actually worked out really well." This was particularly true of actuators commonly placed in the seam hidden under the arm (Figure 6).

The most difficult part of constructing the garment was the interlocking tab system both in terms of the orientation of the tabs and the dexterity needed to connect the tabs, as mentioned by all 13 groups. G9 mentioned that the hardest part of the tab was "just being able to get it into the hole and manipulating. I'm missing part of my finger so I don't have much dexterity, and he has a problem with his finger. So, we are both kind of limited dexterity." Squares also needed to be oriented in a certain way so that the "gender" of the tabs would connect. If this was not thought out ahead of time individuals sometimes ended up with squares that would not connect. G2 "They don't always match up" and G10 "all sides aren't the same so that you can't connect some pieces." This was a result of our $3 \times 3$ and $6 \times 3$ squares, which had an odd number of tabs and therefore could only connect in specific orientations.

To deal with the interlocking tab issues five groups suggested other materials or made requests for future iterations. To solve the orientation issue one group of participants suggested a design for a "single-gender" tab system where all tabs could interlock with each other. For addressing dexterity issues four groups suggested Velcro as an easy way to connect squares. Other suggestions included sewing snaps, buttons, safety pins or long tweezers to pull tabs through.

\subsection{Wearing Different Hats}

Throughout the prototyping process, we told groups to assign one individual to be the "wearer" (W) who wore the garment and the other to be the "non-wearer" (NW) who did not wear the garment. The purpose of these roles was to encourage individuals to consider what it was like to wear the prototype, what it was like to have someone put the Wearable Bits together on their body, and what it was like to place Wearable Bits on someone else.

This role division resulted in several repeating behaviours that we noticed among the groups. The most prominent behavior was that the garment was designed for the wearer. In five groups the teams discussed how the non-wearer measured the wearer with the squares in order to determine the size of the garment. G1 NW "We made the sizes for her. We had to see and adjust on the wearer." G10 W "We wanted it to be a good fit but not too tight... So as we like put it on me we just figured that out." The wearer also determined the design when it came to modesty and comfort. In G2 the non-wearer wanted to add more colours but the wearer disagreed, "I don't like that... I like to blend in... to be very subtle, to figure out what's going on." The wearer also made decisions about aesthetics. G6 W "I wanted to make sure it was all flat, no bumpy stuff." Whereas other wearers were more comfortable adding colours and components. G5 W "I'm used to making a fool of myself so it was ordinary [giggles]”.

Having the wearer role also helped the group to evaluate and test whether their garment was working. Five groups discussed how they evaluated the garment through trying it on. G8 W "You can't figure out the flaws until you've

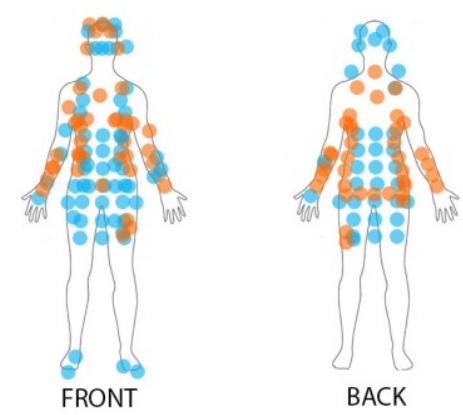

Figure 6-Location of sensors (blue) and actuators (orange) in the front and back 
done that. You have to be able to do it." G3 W "Since I'm the user I can notice the flaws." They could also evaluate the garment before it was complete. G9 W "It gave us that flexibility of adjusting, so that was good." G3 NW "I think it was easy to see how the prototype was going." G8 NW "It certainly helps you think about where pieces need to go. We moved almost everything except the heat sensor." Teams could also evaluate whether their prototype achieved the goals they set out for themselves. G2 NW"The cheer you up dress-you're cheerful now! So, I think it worked!" to which G2W responded "Ya! I feel happy."

Six teams discussed how they used delegation for building. Often one individual found the tabs easier to work with than the other and that became their job. This occurred to an even greater extent if it was the non-wearer that found it easier to connect the tabs. G13 W "You [NW] were faster at plugging things together. So, I just stood there as a template after a while. I worked more as a mannequin than anything else." With the smaller sleeves and accessories often both groups members would try them on while they were building, so even though they had one person who was the wearer they both had moments where they acted as one in order to connect pieces. There was also some sharing of techniques. Two groups specifically mentioned while building that they enjoyed learning about how their partner dealt with the material and their construction techniques.

Several individuals who ended up designing garments that went across the body self-selected to be the non-wearer because of the amount of material they would need and suggested that their partner should be the wearer because of their smaller size. One participant suggested that they would have otherwise wanted to be the wearer. G2 NW "As the non-wearer it was kind of frustrating. It was the better choice or we would have had to use twice the amount of material." A similar sentiment was echoed by G6 NW at the very beginning of the prototyping process when they were deciding roles. This suggests that larger pieces of material are needed so that all individuals who want to be the wearer do not exclude themselves from specific roles based on limitations from the kit.

\subsection{Building on the Fly}

In the questionnaires, 21 of 26 participants agreed that Wearable Bits helped them build on the fly, and all groups mentioned this characteristic in their presentations.

When we started each session, we told the groups not to feel limited by the items in the kit, and if they wanted to add a component they could do so. As a result, 11 of the 13 groups added their own components to the kit by adding an accessory or by writing the name of a component on a blank square. The most common components that were added were temperature sensors (4), zipper sliders (3), and others included a global positioning system (GPS), galvanic skin response (sweat sensor), infrared (IR) sensor, radio frequency identification (RFID) sensor, a receiver, cooling actuation, OLED displays and a Bluetooth module.

Seven groups mentioned that as they began building their prototype they started to add more features and components. G7 "We were building it and as I started going through these [points to bins of interactive components], more and more ideas popped into my head." G10 "We had the base idea and then we expanded on it." Groups also altered their idea along the way. G8 "We had the tilt for motion, but we decided that the stretch would be better." G 12 "We dropped a couple of components." The type of garment the teams decided to build also changed. Two groups started out with jackets concepts that turned into vests.

Two medium fidelity groups prototyped modular e-textile designs that could change what part of the body they were attached to by replacing different pieces. G9 "This is a good tool to do a lot of brainstorming with a person so that you can just do it in a meeting versus sew that together and then coming back. This actually is a more fluid creative process." G12 "Maybe you can actually put it on your leg, hang it on your backpack, moving it around a bit, that would be cool." These two examples demonstrate how the medium-fidelity prototypes could enable groups to prototype modularity into their final design. This design direction did not occur in the low-fidelity prototypes.

\subsection{Envisioning Future Aesthetics}

At the end, groups presented thoughts on their garment's materials, colours, and the invisibility of the technology. Since all the individual pieces of Wearable Bits were made of felt, materials were one aspect of the design that often did not come up during their prototyping process until their final presentations. When they were asked about what they thought of their final prototype, nine groups envisioned specific materials (such as G11 "denim or canvas") or material properties (such as G7 "swimsuit kind of material, sporty, maybe stretchy") for their wearable garment. Five groups discussed wanting stretch or elasticized materials, and two groups wanted knitted material (one for a hat and one for a glove/sleeve). At times material choices had to do with glamour or the price point of the material. G2 "This dress, although it looks like it's 
made out of felt, is actually very expensive fringing." G4 "I could see if it was for rockers, like a black sueding type thing, and lots of glitter." Overall the participants had clear design ideas about how they envisioned their final garment that would not have been explored without the final presentation questions. This is one limitation of the Wearable Bits toolkit and indicates that more materials should be included in the toolkit or that researchers using the toolkit need to have material discussions with participants.

Another limitation that came up in the final presentation was participants either running out of colours they liked or imagining different colours. Five groups discussed colours they would have preferred to incorporate into their prototype. G1 ran out of the colour they started with and resorted to using other colours, "these black pieces were supposed to be grey." G3 "I wouldn't use four different colours. Something that is pretty and fashionable." Sometimes colour was a dealbreaker and this highlighted the importance of colour in the prototyping process. When canvas came up as the material of choice, G11 emphasized that it could not be beige canvas, "If this thing was an ugly beige there's no way I would buy it. Even if you told me it lit up."

All groups chose the type of garment based on the context of the activity, such as gym shorts for running and pajamas for sleeping. Items that were meant to be worn regularly in public became accessories such as the three groups that prototyped hats. All but one group emphasized that the garment should match the context. The one group that did not do this made an activism wearable that was meant to look invisible most of the time, but then would grab attention to encourage discussion at specific moments. Four groups emphasized that they wanted the technology to be hidden behind or within other fabrics. G2 "The sensors are under the fringing-Note! [Pointing at the researcher to write that down]." G5 "I would imagine if you were using yarn of some kind that it could be embedded that you would knit and build your design that way." One group that made a wearable for arthritis emphasized that it should not be "medical looking". G8 "Not at all. More like a ski glove kind of thing." Participants mentioned that with lighting effects a material to diffuse the lighting was important. G9 "It could be very elegant. [...] This would be an undergarment that could be enhanced with puffy sleeves that are backlit like a sheer."

\section{DISCUSSION}

Our approach of tangiblizing e-textile components into physical 'Bits' stems from TEI's fundamental concepts of
'Radical Atoms' [21] and 'Tangible Bits' [20, 22] where HCI bridges the gap between the digital and the physical. In this sense, our Wearable Bits offered non-expert users a tangible method of envisioning and designing their own wearable interfaces. Despite being low and medium fidelity, the Wearable Bits empowered 26 people to design their own wearables and scaffold their creativity. The use of lowcost materials and tools also supported accessibility and paved the way for further research to engage different groups of people in wearable technology in various contexts (such as school children, senior care homes, etc). Giving such groups a voice-through using Wearable Bitsbrought new insight, potentials and opportunities that we, as designers, would not have developed otherwise.

One of the key differences between our Wearable Bits and other e-textile toolkits is that it focuses on creativity and enabling strategies rather than getting entangled in the feasibility and skillset that are all usually entailed in other toolkits. Relevant work [10, 23, 38] mostly require fine crafting and/or technical skills, or workshops to acquire those skills, to create small swatches or samples of what is possible. Alternatively, all our participants have used Wearable Bits to design and create their wearable garments in 1:1 real-world scale (including a dress, a jacket, pants, shorts, a vest, a crop top, a sleeve and a hat). In addition, they all expressed how it was "fun", "easy to use" and "fast" in how the kit supported rapid prototyping.

\subsection{Moving Beyond Fitness Trackers}

Of the 13 prototypes developed during our study only 1 group made a fitness device, and only 1 group made a medical or health-related device. The groups that did monitor bodily signals mostly used them for selfexpression, such as the pregnancy shirt that expressed to the outer world what was going on inside the womb. As G13 described their reasoning, "Originally, we were looking at a monitoring aspect, but you're already keeping a really close eye on that sort of thing anyhow, and this was more outwardly expressive, which makes it a little bit more interesting." Using biosignals as expressive cues rather than for health and fitness has been documented by Howell et al. [17] and e-textile garments for expressive rather than health and fitness purposes in the work of Davendorf et al. [10]. The wide variety of garment concepts developed with Wearable Bits runs in this same vein, demonstrating that individuals want wearable e-textiles for reasons beyond those currently commercially available. 


\subsection{Locating Interactivity in Garment Design}

There is much prior work on socially acceptable locations on the body for wearable technology $[42,50]$. Our findings differ in that our participants expressed more concern about how well the technology was integrated into the design of the garment rather than on how socially acceptable a specific location was. For example, the most common location for actuators in our study was in the seam area below the arm and the seam area in the shoulder, which were not common locations in previous work. This was also supported by participants wanting the technology to be so well integrated into the garment it was invisible.

This design approach of ensuring that the technology fit with the design of the garment is best demonstrated in the prototype developed by G2. They designed a flapper dress where the sensors (stroke sensors) acted as the fringe in the entire midline and upper leg area in the front and back. They went through the motions of stroking these areas around the midline, upper leg and buttocks area, and thought that this action integrated well with the garment. They also prototyped speakers in the bra area because of how well the circular design of e-textile speakers would fit with the design of that area of the dress. This example, and the many groups that prototyped technology along the seams, demonstrates how individuals might desire wearable technology in areas previously thought of as social unacceptable as long as it is well integrated into the garment design. Previous work has embedded sensors into seams such as StretchEBand [48], or embedded technology into embroidery designs [13]. The importance of integrating technology into the design of an e-textile garment has also been highlighted in previous work on etextile actuation [10].

This difference in our location findings to those of previous work could be due to the nature of our toolkit where individuals can make larger sweeping interactions, rather than previous work such as JogWheel [42] where individuals are seeing a video of someone interacting with or "poking" very specific locations on the body. The overall benefit of our evaluation method is that the prototypes are evaluated from two perspectives: that of an embodied wearer and that of an embodied viewer who is seeing the prototype being used in front of them at life scale. Yet individuals are not in public with their prototypes on and this more public evaluation of social acceptability is an important area for future research.

\subsection{The Importance of a Wearer}

In the garment industry, it is common to prototype garments on body forms or mannequins where toile is the prototype the designer makes out of cheaper fabric such as muslin [43]. This tradition of prototyping on mannequins has carried over into e-textile prototyping tools such as Mannequette [45]. In contrast, our work demonstrates the importance of prototyping with a wearer rather than a mannequin when working with novice users such as in codesign. We found that the benefits of this approach include considerations for social acceptability and comfort, considerations for the wearer's body shape and measurements, and enabling the wearer to act as an evaluator or tester for the prototype. Having a wearer often led to changes in the garment prototype as groups explored ideas and reevaluated their garment concept. When we consider the other e-textile prototyping toolkits such as card sorting [34] and swatchbooks [15, 39], this is the benefit of a toolkit like Wearable Bits. By placing the components on the body wearers must consider what that feels like. This would be another benefit of an e-textile constructive assembly [1] where individuals can go a step further and test out not only what it feels like to wear certain sensors and actuators, but what the interactions themselves would feel like.

\subsection{Insights for Future Prototyping Toolkits}

In the Wearable Bits workshops, we discovered several areas of insights for future e-textile toolkits.

Design for collaboration: We found that having a wearer, and encouraging discussion by having a collaborator, were valuable components of thinking through the design while building it. The wearer was able to evaluate how it felt to wear the design and the non-wearer was able to provide feedback on how it looked from the outside. This collaborative learning through doing approach allowed the team to evaluate the resulting prototype from these two important viewpoints. The design of the toolkit also allowed for this collaboration because more than one individual could be working on it at one time, adding components or replacing parts.

Expand materials: The most significant gap in the toolkit was material exploration, since all pieces were laser cut felt. In their presentations, participants had clear ideas about materials that they would like to use in their envisioned garments. This suggests that we need a way for users to express and explore material options. In their review of etextile toolkits Posch et al. [40] also found a scarcity of material exploration in this area of research, often in the 


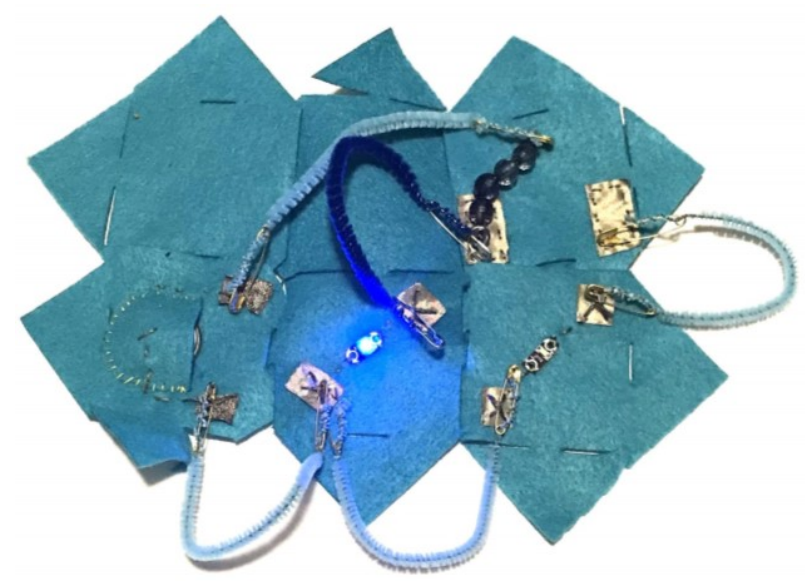

Figure 7-A simple circuit made with the medium-fidelity bits, including a tilt sensor that lights up.

name of making toolkits easier to use. Our paper highlights how material exploration is important not just to e-textile designers, but also to end-users of these toolkits.

Enable current building techniques: We found that users often began by building the garment with the larger squares and wanted them even larger. Larger bits would allow individuals to spend much less time connectin ' $g$ tabs and make it easier to build garments that require more fabric such as dresses and cloaks. Having larger squares to work with would also be more inclusive of the diversity of possible body sizes. A second building technique was that participants often built the interactive bits on top of their base garment as a second layer. Our current toolkit was a bit clumsy for with this type of interaction and we will work on making it easier to build using a second layer.

Choose the right toolkit for the design stage: The benefit of our low-fidelity bits was that users felt free to mark them up, glue down components, and get crafty. This type of flexibility is especially useful for early stage designs, but this craftiness did not occur with the medium-fidelity bits, and instead two groups incorporated the modularity of those components into their final design. Our next step is to build Wearable Bits into a constructive assembly for prototyping the interactions [25], but it is also important to consider what we could "lose" from a constructive assembly. We predict that users will feel less free to mark them up as they did with the medium-fidelity bits. Therefore, we suggest changing the toolkit based on the prototype stage. One could start with the low-fidelity bits, then replace them as the fidelity increases and ideas are solidified. Our study suggests that there is no one ideal toolkit, but rather toolkits suitable for specific design stages.

\section{CONCLUSION}

The purpose of a prototype is to iteratively improve upon a design and explore options. In co-design with end-users the purpose of a toolkit is to scaffold creativity and help participants to express their ideas [44]. In this paper, we contributed a co-design toolkit called Wearable Bits for prototyping wearable e-textiles and evaluated it with 26 workshop participants. Except for with material and fabric exploration, Wearable Bits enabled participants to express their wearable e-textile concepts and work on the fly. Groups developed a wide variety of concepts and the toolkit had enough flexibility that individuals could add their own components and accessories. We demonstrated the value of prototyping garments on a wearer and discuss how researchers can improve upon Wearable Bits in future work. Our aim is that Wearable Bits can continue to broaden the design space for e-textiles and enable endusers to participate in the design of their own garments to suit their own needs.

\section{ACKNOWLEDGEMENTS}

We would like to thank all our participants who generously contributed their time and ideas to this study. We would also like to thank our reviewers for their insights that helped to improve this work. This work was supported and funded by the National Sciences and Engineering Research Council of Canada (NSERC) through a Discovery grant (2017-06300), a Discovery Accelerator Supplement (2017507935), by the Ministry of Ontario through an Early Researcher Award (ER15-11-101). The views expressed in the publication are the views of the Institution and do not necessarily reflect those of the Province.

\section{REFERENCES}

[1] Bdeir, A. 2009. Electronics as material: littleBits. Proceedings of the Third International Conference on Tangible and Embedded Interaction (TEI'09), (2009), 371.

[2] Berber Soepboer and Fioen van Balgooi - Fragment Textiles: 2009. https://en.refinity.eu/fragment-textiles.html.

[3] Boone, A., Rivera, E. and Wolf, J. 2018. Patchwork: an expressive etextile construction kit. Proceedings of the 17th ACM Conference on Interaction Design and Children - IDC '18 (2018), 529-532.

[4] Buchenau, M. and Suri, J. 2000. Experience prototyping. Designing Interactive Systems (2000), 424-433.

[5] Buechley, L. 2007. A construction kit for electronic textiles. Proceedings - International Symposium on Wearable Computers, ISWC (2007), 83-90.

[6] Buechley, L., Eisenberg, M., Catchen, J. and Crockett, A. 2008. The LilyPad Arduino: Using Computational Textiles to Investigate Engagement, Aesthetics, and Diversity in Computer Science Education. Proceeding of the twenty-sixth annual CHI conference on Human factors in computing systems - CHI '08 (2008), 423.

[7] Buechley, L., Elumeze, N., Dodson, C. and Eisenberg, M. 2005. Quilt snaps: A fabric based computational construction kit. Proceedings IEEE International Workshop on Wireless and Mobile Technologies in Education, WMTE 2005 (2005), 219-221.

[8] Buechley, L. and Hill, B.M. 2010. LilyPad in the wild: how 
hardware's long tail is supporting new engineering and design communities. Dis 2010.

[9] Circular Open Source Fashion: https://class.textileacademy.org/classes/week03/.

[10] Devendorf, L., Lo, J., Howell, N., Lee, J.L., Gong, N., Karagozler, M.E., Fukuhara, S., Poupyrev, I., Paulos, E., Ryokai, K. and Berkeley, U.C. 2016. "I don't want to wear a screen ": Probing Perceptions of and Possibilities for Dynamic Displays on Clothing. CHI '16: Proceedings of the 2016 CHI Conference on Human Factors in Computing Systems (2016).

[11] Fitzmaurice, G.W., Ishii, H. and Buxton, W. 1995. Bricks: laying the foundations for graspable user interfaces. Conference on Human Factors in Computing Systems - Proceedings (1995), 442-449.

[12] Gilliland, S., Komor, N., Starner, T. and Zeagler, C. 2010. The textile interface swatchbook: Creating graphical user interface-like widgets with conductive embroidery. Proceedings - International Symposium on Wearable Computers, ISWC. Figure 1 (2010), 1-8.

[13] Hamdan, N.A., Voelker, S. and Borchers, J. 2018. Sketch\&Stitch: Interactive Embroidery for E-Textiles. Proceedings of the $2018 \mathrm{CHI}$ Conference on Human Factors in Computing Systems - CHI '18 (2018), $1-13$.

[14] Hartmann, B., Klemmer, S. R., Bernstein, M., Abdulla, L., Burr, B., Robinson-Mosher, A., \& Gee, J. 2006. Reflective Physical Prototyping through Integrated Design, Test, and Analysis. Proceedings of the 19th annual ACM symposium on User interface software and technology (2006).

[15] Hertenberger, A. et al. 2014. 2013 E-Textile Swatchbook Exchange: The Importance of Sharing Physical Work. Proceedings International Symposium on Wearable Computers, ISWC (2014), 7781.

[16] Hornecker, E. and Buur, J. 2006. Getting a grip on tangible interaction: A framework on physical space and social interaction. Proceedings of the SIGCHI conference on Human Factors in computing systems (2006), 437-446.

[17] Howell, N., Devendorf, L., Tian, R. (Kevin), Vega Galvez, T., Gong, N.-W., Poupyrev, I., Paulos, E. and Ryokai, K. 2016. Biosignals as Social Cues: Ambiguity and Emotional Interpretation in Social Displays of Skin Conductance. Proceedings of the 2016 ACM Conference on Designing Interactive Systems - DIS '16. (2016), 865870.

[18] Hummels, C. and Van Dijk, J. 2015. Seven principles to design for embodied sensemaking. TEI 2015 - Proceedings of the 9th International Conference on Tangible, Embedded, and Embodied Interaction (2015), 21-28.

[19] Hur, E.S. and Thomas, B.G. 2011. Transformative Modular Textile Design. Bridges 2011: Mathematics, Music, Art, Architecture, Culture. (2011), 217-224.

[20] Ishii, H. 2008. Tangible Bits: Beyond Pixels. Proceedings of the 2nd international conference on Tangible and embedded interaction (2008).

[21] Ishii, H., Lakatos, D., Bonanni, L. and Labrune, J.-B. 2012. Radical Atoms: Beyond Tangible Bits, Toward Transformable Materials. Interactions.

[22] Ishii, H. and Ullmer, B. 1997. Tangible bits: towards seamless interfaces between people, bits, and atoms. Proceedings of the ACM SIGCHI Conference on Human factors in computing systems (1997), 3-3.

[23] Jelen, B., Freeman, A., Narayanan, M., Sanders, K.M., Clawson, J. and Siek, K.A. 2019. Craftec: Engaging Older Adults in Making through a Craft-Based Toolkit System. Proceedings of the Thirteenth International Conference on Tangible, Embedded, and Embodied Interaction - TEI '19 (2019), 577-587.

[24] Jones, L. 2019. A co-design toolkit for wearable e-textiles. Proceedings of the 2019 ACM International foint Conference on Pervasive and Ubiquitous Computing and Proceedings of the 2019 ACM International Symposium on Wearable Computers (2019), 363366.

[25] Jones, L., Nabil, S. and Girouard, A. 2020. Swatch-bits: Prototyping E-textiles with Modular Swatches. Proceedings of the Fourteenth
International Conference on Tangible, Embedded, and Embodied Interaction (2020).

[26] Jun Kawahara Kimono ++: https://class.textileacademy.org/2019/jun.kawahara/projects/final-project/.

[27] Kazemitabaar, M., Mcpeak, J., Jiao, A., He, L., Outing, T. and Froehlich, J.E. 2017. MakerWear: A Tangible Approach to Interactive Wearable Creation for Children. Proceedings of the 2017 CHI Conference on Human Factors in Computing Systems (2017), 133-145.

[28] Kazemitabaar, M., Norooz, L., Guha, M.L. and Froehlich, J.E. 2015. MakerShoe: Towards a Wearable E-Textile Construction Kit to Support Creativity, Playful Making, and Self-Expression. Proceedings of the 14th International Conference on Interaction Design and Children (2015), 449-452.

[29] Kettley, S. 2016. Designing with Smart Textiles. Bloomsbury Publishing.

[30] Klemmer, S.R., Hartmann, B. and Takayama, L. 2006. How bodies matter: Five themes for interaction design. Proceedings of the Conference on Designing Interactive Systems: Processes, Practices, Methods, and Techniques, DIS (2006), 140-149.

[31] Knapp, J., Zeratsky, J. and Kowitz, B. 2016. Sprint: How to Solve Big Problems and Test New Ideas in fust Five Days. Simon and Schuster.

[32] Kuznetsov, S., Trutoiu, L.C., Kute, C., Howley, I., Siewiorek, D. and Eric Paulos 2011. Breaking Boundaries: Strategies for mentoring through textile computing workshops. Proceedings of SIGCHI(2011).

[33] Leong, J., Perteneder, F., Jetter, H.-C. and Haller, M. 2017. What a Life! Building a Framework for Constructive Assemblies. Proceedings of the Eleventh International Conference on Tangible, Embedded, and Embodied Interaction (2017), 57-66.

[34] Nevay, S. and Lim, C.S.C. 2015. The Role of Co-Design in Wearables Adoption. Contemporary Ergonomics and Human Factors 2015: Proceedings of the International Conference on Ergonomics \& Human Factors 2015, Daventry, Northamptonshire, UK, 13-16 April 2015 (2015), 109

[35] Ngai, G., Chan, S.C.F., Cheung, J.C.Y., Kong, H. and Lau, W.W.Y. 2009. The TeeBoard: an Education-Friendly Construction Platform for E-Textiles and Wearable Computing. SIGCHI Conference on Human Factors in Computing Systems (2009), 249-258.

[36] Ngai, G., Chan, S.C.F., Leong, H.V. and Ng, V.T.Y. 2013. Designing iCATch: A multipurpose, education-friendly construction kit for physical and wearable computing. ACM Transactions on Computing Education (TOCE). 13, 2 (2013), 1-30.

[37] Park, J., Morris, K., Stannard, C. and Hamilton, W. 2014. Design for many, design for me: Universal design for apparel products. Design Fournal. 17, 2 (2014), 267-290.

[38] Perner-Wilson, H., Buechley, L. and Satomi, M. 2011. Handcrafting Textile Interfaces from A Kit-of-No-Parts. Proceedings of the fifth international conference on Tangible, embedded, and embodied interaction (2011), 61-68.

[39] Posch, I. and Fitzpatrick, G. 2018. Integrating Textile Materials with Electronic Making: Creating New Tools and Practices. Proceedings of the Twelfth International Conference on Tangible, Embedded, and Embodied Interaction - TEI'18 (2018), 158-165.

[40] Posch, I., Stark, L. and Fitzpatrick, G. 2019. eTextiles: Reviewing a Practice through its Tool / Kits. Proceedings ofthe 2019 International Symposium on Wearable Computers (ISWC '19 (2019), 195-205.

[41] Post, E.R., Orth, M., Russo, P.R. and Gershenfeld, N. 2010. Ebroidery: Design and fabrication of textile-based computing. IBM Systems fournal. 39, 3.4 (2010), 840-860.

[42] Profita, H.P., Clawson, J., Gilliland, S., Zeagler, C., Starner, T., Budd, J. and Do, E.Y.-L. 2013. Don't mind me touching my wrist: A Case Study of Interacting with On-Body Technology in Public. International Symposium on Wearable Computers (2013), 89-96.

[43] Rissanen, T. and McQuilla, H. 2016. Zero Waste Fashion Design. Bloomsbury.

[44] Sanders, E.B.N. and Stappers, P.J. 2014. Probes, toolkits and prototypes: Three approaches to making in codesigning. CoDesign. 10, 1 (2014), 5-14.

[45] Seyed, T. and Tang, A. 2019. Mannequette: Understanding and 
Enabling Collaboration and Creativity on Avant-Garde FashionTech Runways. Designing Interactive Systems (2019).

[46] Smit, D., Oogjes, D., De Rocha, B.G., Trotto, A., Hur, Y. and Hummels, C. 2016. Ideating in skills: Developing tools for embodied co-design. TEI 2016 - Proceedings of the 10th Anniversary Conference on Tangible Embedded and Embodied Interaction (2016), 78-85.

[47] Tomico, O. and Wilde, D. 2015. Soft , Embodied, Situated \& Connected. Proceedings of the 17th International Conference on Human-Computer Interaction with Mobile Devices and Services Adjunct (2015).

[48] Vogl, A., Parzer, P., Babic, T., Leong, J., Olwal, A. and Haller, M. 2017. StretchEBand: Enabling Fabric-based Interactions through Rapid Fabrication of Textile Stretch Sensors. SIGCHI Conference on Human Factors in Computing Systems (2017), 2617-2627.

[49] Wilde, D., Vallgårda, A. and Tomico, O. 2017. Embodied design ideation methods: Analysing the power of estrangement Conference on Human Factors in Computing Systems - Proceedings (2017), 5158-5170.

[50] Zeagler, C. 2017. Where to Wear It: Functional, Technical, and Social Considerations in On-Body Location for Wearable Technology 20 Years of Designing for Wearability. Proceedings of the 2017 ACM International Symposium on Wearable Computers (2017), 150-157.

[51] Zeagler, C., Audy, S., Pobiner, S., Profita, H., Gilliland, S. and Starner, T. 2013. The electronic textile interface workshop: Facilitating interdisciplinary collaboration. International Symposium on Technology and Society, Proceedings (2013), 76-85. 